\title{
Information Search habits of first year college students
}

\author{
Higinio Mora-Mora ${ }^{1, *}$, María Teresa Signes-Pont ${ }^{1}$ and Gregorio De Miguel Casado ${ }^{2}$ \\ ${ }^{1}$ Department of Computer Technology and Computation, University of Alicante, Campus de San Vicente del \\ Raspeig, 03690 Alicante, Spain. E-mail: \{hmora, teresa\}@dtic.ua.es \\ ${ }^{2}$ Department of Computer Science and Systems, University of Zaragoza, 50009 Zaragoza, Spain. E-mail: \\ gmiguel@unizar.es \\ * corresponding author. E-mail: hmora@ua.es
}

\begin{abstract}
.-
New technologies have transformed teaching processes and enabled new ways of study and learning. In these activities, it is suspected that the students don't make good use of new available technologies or, in the best case, they are underused. The analysis of this issue with the design of strategies to correct any defects found is the motivation that supports the development of this work and the main purpose of it. Evaluate information search habits used by the student and analyse their deduct synthesis and processing capabilities of the results found. The researchers of this study are university teachers of first year subjects, which allows them to know the information search performances by students.
\end{abstract}

Keywords.- Student search habits, teaching and learning processes, new technologies and internet.

\section{Introduction.}

The fast development of the computing technologies and of the information and communication networks have made possible that the teaching processes have experienced many changes that have allowed new ways of study and learning of all subjects.

Since some years ago, on the first year subjects form the Degree on Computer Science from the University of Alicante, it has been implemented a continuous evaluation system on which is common the development of many test or practical assignments that are submitted during the semester (four-month period). The grade is added to a final theoretical and practical test performed before the ending of the semester. Usually, some of these tests consist on the performance of a desk research on a particular aspect from the subject with the objective of extend or complete its content. On it's performing; students have to proceed with the information research about the topic entrusted. Without having to perform other analysis, a first lecture shows a large lack of rigor of the delivering and an overwhelming source coincidence.

Before the emergence of the Internet, the information research was limited to the review of books or papers specialized in the field. Nowadays Internet is present in our lives, that network of networks contain unlimited information about almost any topic. However, not all is correct and precise. Is necessary to know how to search, compare and select the suitable information on each situation. This task is not as easy as the student think, and in fact, constitutes one of the fundamental objectives of the subject and of the production process performed by the student. 
The researcher team of this issues are teachers of first year university courses. They have extensive teaching and research experience in the fields of innovative educational methods (Mora, 2009), (García et al., 2012). To analyse the habits of the students on information research we count with the accumulated experience of the teachers mentioned at the revision of documentation works and practices of this kind, and in addition, with qualitative information obtained by means of an anonymous survey to all the students where they were asked about the technological and bibliographic resources used on the information research as in the production process of the researching assignments. The analysis of the previous results will allow identifying the deficiencies and problems of the students on this process as well as direct the efforts to learn about how to use the new technologies to the knowledge location on an efficient way.

The remainder of the paper is organized as the following: section 2 presents the background and related works. Section 3 defined the methodology used to find out the search habits students have. Section 4 illustrates the main results and, finally, section 5 concludes the paper.

\section{Related work}

There are many previous studies that analyse the researching processes on the Internet by the users, both at university as non-university. These projects try to extract the keys that remark the differences between the time spent on the research, used filters and number of queries performed (Spink \& Jansen, 2004), (Efthimiadis, 2009), (Hess, 1999), (Hwang et al., 2007). Some of the conclusions of these studies are discussed below. The big amount of information abatable on the Internet requires of a certain quantity of ability to obtain the expected results. One of the key aspects to success on this task is having the mental and conceptual schemas necessaries to orient the research with the proper key words (Brandt, 1997), (Uden et al., 2001). These characteristics increase basically based on the experience, and with it, the results are simplified and increases the efficiency in the process (Perrochon, 1997), (Munro \& Kwon, 1997), (Newsweek, 2010), (Song \& Salvendy, 2003).

About the evaluation of the found information, the interest aspects must focus on ensuring which found material is error-free, author trust, objective and current (Stenger \& Goode, 1998), (Tsai, 2003). In relation to the university students, a special searching type is the one that is related with their studies. Respect from that, there are many studies that pretend to evaluate the degree of satisfaction and facility of the webpage of the departments from the universities to offer their relevant information: teachers, schedules, credits of the subjects, etc. (Jacobson and Cohen, 1997), (Pointdexter \& Heck, 1999), (Johnson, 2003), (Palmer \& Kent, 2007).

Another basic and innovative aspect is the use of the social networks to the information exchange. This is a way of information that is in continuous growth and development and has many acceptation by the students. There are many investigation projects about the way of treating and disseminate this information (Zhuhadar, 2013), (Lytras et al., 2014), (Mora et al., 2014). Recent studies about the diffusion of the information in blogs with respect to other social networks conclude that blogs are being a huge source to transmit the information, having these a considerable amount of inputs compared to other type of webs with specialized information (Tang et al., 2009).

As a conclusion of this short review of previous works we obtain that the topic of this study is also focused on a big amount of investigations by its relevance on different fields. Traditionally the social and economic fields try to collect the keys that 
improve the positioning and visibility of a information or web; The technological field tries to provide and improve tools (search engines, metasearches, etc.) that simplify this task; while, on the other hand, the academicals aspects try to teach using these resources properly.

On this job we are going to focus the attention on students of the Computer Engineering degree at early courses, due to the fact that there aren't previous studies about them. We will depart from the premise that these users are going to be more used with the use of Internet and the on-line resources to search information. We are going to observe their searching habits in order to extract conclusions about some aspects as: witch searching engines they use, sources, language and the way of evaluating and synthesize the contents to its following processing.

\section{Methodology.}

\subsection{Subjects of study.}

In our research we will select a group of students (52 students) of first year of Computer Engineering Degree of the University of Alicante. These students have an age between 18 and 20 years old.

\subsection{Procedure.}

In order of being able to recognize the information of the students we will make an anonymous survey that they will fulfil and deliver to the professor. This survey has been performed the last week of class of the course. The survey is composed by 38 questions (4 answer options each) grouped in 5 categories:

1. General: Computers, mobile phones, Internet connexion, operative system, Internet browser, text processor, etc.

2. Social Internet: blogs, instant messaging and social networks.

3. General information search: search bar, search engine, information searching places, searching time, preferred language, filters, suggestions, information synthesis, etc.

4. Specialized information search: general information websites, searching places, scientific searchers, university webs, etc.

5. Rigour and exactitude on the information search: information veracity, contrast information, reliable information, demonstrations, etc.

Most of questions only allowed choosing one answer, the most used, although there are many multiple-choice questions.

\section{Results.}

Now are going to be analysed some of the answers obtained on relevant questions to the objectives of this project.

\subsection{General information.}

This collection of questions, pretend to obtain general information of the student's technological point of view. As result, we know that most of them own both laptop and 
desktop computer $(72 \%)$, has a $3 \mathrm{G}$ mobile phone $(65 \%)$ and has internet connection at the computer (61\%), also, 39\% remaining has internet on the mobile phone and the computer. Regarding to the software installed on the working computer (not mobile phone or tablet), we know that $78 \%$ of them uses Windows $8 / 7 /$ Vista, a $17 \%$ uses some Linux distribution and the 5\% has Mac OS. The most used web browser is Mozilla Firefox with a $72 \%$, and the most used text processor is Microsoft Word with an $83 \%$ while Open/libre Office stays in the back with a 17\%. It has to be taken into account that due to the fact that we are talking about Computer Engineering students exists a high rate of UNIX based systems installed because it is a widely used tool in those studies.

Almost all students have home access to the Internet and broadband networks. Nevertheless, they prefer the university facilities as a place of study and performing the documentation works.

\subsection{Social Internet.}

First of all, we asked the student what understands by "social internet". As answers we found that the most common where those about communication between persons and social interaction through social network over internet: common web platforms are: Twitter, Google+ or Facebook. Some responses also include applications like blogs and chats. Students refer to all of this as Web 2.0.

Instant messaging tools are used by $100 \%$ of the survey respondents. Most of them is registered in Windows Live Messenger, although Messenger is being taken out of place by the new instant messaging tools incorporated on the social networks like Twitter or Facebook and other integrated on mobile devices as WhatsApp. About Social Networks usage, the next figure shows the percentages of students that have an account or profile created and access frequently.

Figure 1. Use of Social Networks among students

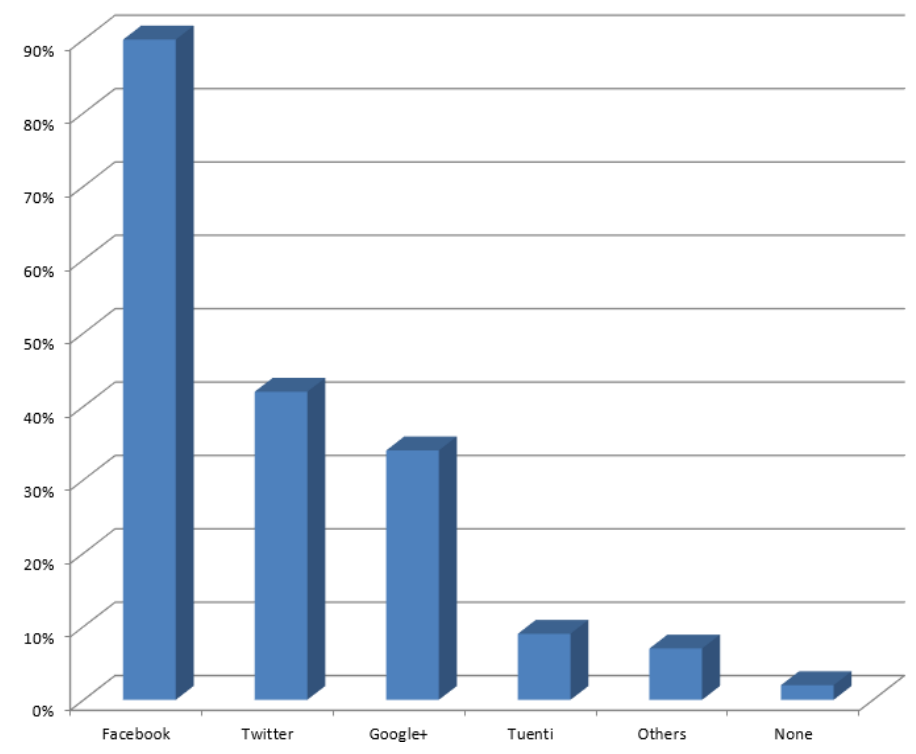

It is observable that the use is generalized between the students and that just $2 \%$ (one student) tells that is not registered in any of them. To the information searching processes, the social network interaction is not really important. For the preparation of documentation works they are not one of the sites where for finding information. 
Otherwise the students recognize that social networks are really useful on their learning tasks, especially those related with the university, subject and professor. The tools that they incorporate allow them to exchange information, opinions and reviews and the possibility of performing questions about aspects of the subject.

With respect to the use of blogs, just $17 \%$ of the interviewees own a blog, and around $77 \%$ of them visit those places in order to find information in internet. When asked about the reason for this preference the answer indicates that the blog usually offer organized and clear information on the subjects of searching works. Moreover, for these students, blogs on computer and technical issues are numerous and consistent with the themes of the works. From experience of the faculty, they have found works that are verbatim content from informative blogs.

\subsection{Global information search}

Respect to the information searching process itself. The searching of information is almost exclusively online (92\%) so just a few (8\%) use notes or previous projects. All the students use Google as default search engine and most of them (72\%) integrates google toolbar on their browser to make easier and more efficient the task.

One of the remarkable questions is related with the procedure: $67 \%$ of the students says that they spend a lot of time searching information, and also continues with this task even having some answers. Other $17 \%$ gives the process by concluded when they found the first answer. From that we can extract two conclusions:

- Most of the students aren't sure with the quality of the information that they are founding and they need from other sources to compare and perform the task.

- Other students, or are sure that the answer that they have found is correct, or they just accept the first option as the correct one. Nevertheless, taking into account the past delivery works it seems that the common option is the second one.

Another important point is relative to the information sources, more specifically which places are preferentially visited. On this situation the survey shows that at least $40 \%$ of the students don't care about which is the found page if it has information related to the searched topic, while the rest present certain attention to the source and founding specialized pages. This result proves that the quality of the results is not really important to the students of first year on the search process. The analysis made of the documentation works delivered by the students prove effectively this fact. On the documents are too many references to non-academic or non-scientific pages, where the rigor is not their main goal (for example: wikis-Wikipedia, personal webs, blogs or forums).

The searched type of information is usually text, but the $56 \%$ says that use to complete it with images and a $36 \%$ with explicative videos.

About the use of filters or advanced search most of the students (67\%) uses just the first results. The rest perform a deeper search until find the most suitable result. For them is important to pay special attention to the suggestions of the search engine and the remarked results. This result comes to confirm the low ability on the process, due to the fact that accessing just to those results you fall on the browser tramp. Because can be accessing to its predefined webs that may be ordered by other interests or commercial criteria, more that for the transcendence of its content.

In order to use more of one search engine, the results are divided: a 59\% often use just their usual search engine and another 39\% uses another on a complementary way just when is just fount a little information about something. Not many students 
know the existence of "Metasearchers" to perform parallel searches on many engines and increase in that way the search range (Abawajy \& Hu, 2005), (Boo \& Anthony, 2011).

In relation with the language of the search, most of the students perform the search in Spanish. Although is one of the languages with higher penetration on the internet (Quirós Romero \& Crespo Galán, 2007), the amount (and quality) of results, especially on computing and scientific fields, is much lower than the generated on the most common language, English. This fact calls specially the attention, because many of the materials and bibliographic references used on their studies are written in English. However, asked about this fact, they defend that even having less results in the Spanish search, it is easier to write down and work with those results if they are directly in Spanish.

The analysis of these results fits with the habits of many students based on copy literally the text from the search results. Obviously the "copy paste" is just possible with Spanish results even that in some situations, the teaching staff have detected a "copytranslate-paste" from an English source.

Finally, regarding the processing of the obtained results, $61 \%$ of the students say that they synthesize or extract the results to redact the final assignment, fact that is not what we observe on the empirical evidence. And also $28 \%$ says that expand it with other sources as books or class notes. That fact evidences a high "dependency" from the Internet on this aspect.

\subsection{Specialized information search}

Asked about the specialized searching process about a subject, students did not differ between generic searches and specialized. In both situations they use a generic engine to do the job.

The question about their knowledge of the existence of scientific repositories and documentation is illuminating for us: when they arrive to the university they don't know about repositories and article and scientific text search engines. The following figure represents this result in function of the degree of popularity of the scientific repositories.

Figure 2 Knowledge about scientific repositories.

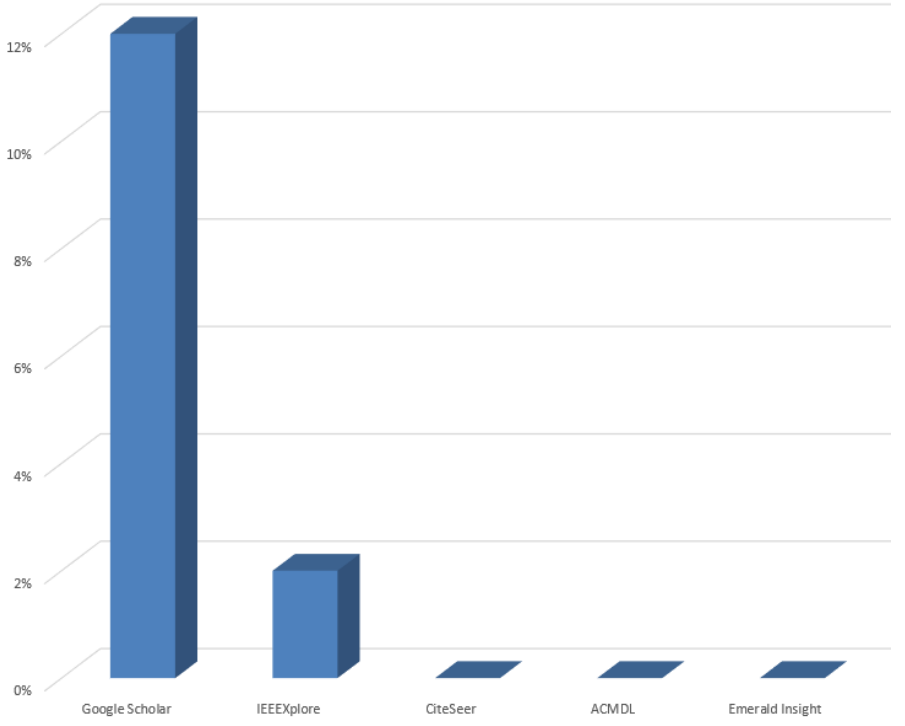


This is one aspect of increased emphasis by the team of teachers. In addition, the University of Alicante is subscribed to the major scientific editorial publishers (IEEE, Elsevier, Springer, etc.) for free web access to content protected by copyright. In this way, it is shown to the students the differences between generic and scientific content searches, and also, the format differences between the two types of documents (personal opinions, formulation, demonstrations, references, etc.).

\subsection{Rigour and exactitude in information search}

The last apart is focused on the credibility that the student gives to the obtained information, and therefore, the rigour that implicitly moves to the writing. This section is closely related to the previous one.

The results are the following: $100 \%$ believes that it is correct when it comes from a scientific text; $95 \%$ when is obtained from some specialized page (for example a page specialized on graphic cards); $92 \%$ when comes from a book, and $90 \%$ when reads it on the Wikipedia; finally $75 \%$ trust the information when comes from a generic page. The following figure shows these results summarized.

Figure 3 Source credibility

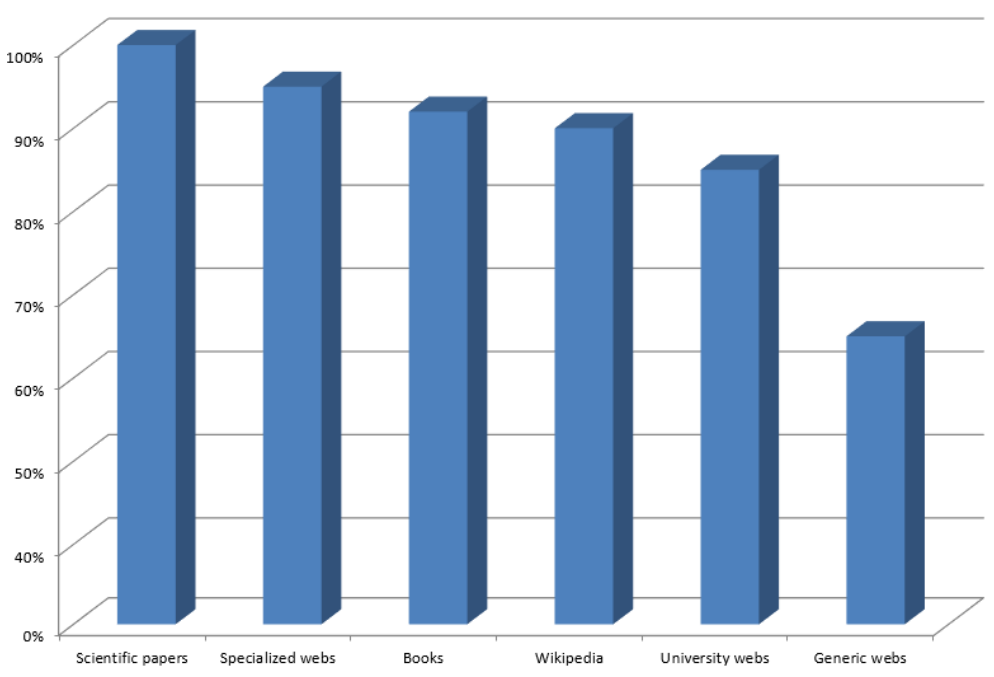

In essence, although they assume different degrees of trust in the information retrieved, the students have much confidence on the contents that they found on the Internet. On the other hand, even taking into account that this is not reflected on the previous data, the degree of the credibility changes with the subject that is going to be searched. On this situation the students admit being more exhaustive with scientific information that requires of some kind of formulation or demonstration than with other kind of contents.

This is the other main target for teaching staff in training for information search in internet. The teaching staff are developing education programs not only to teach the search procedure (language, diversity, metasearchers, etc), but to encourage them to think critically and analytically when they compose their documentation works.

From the first moment, the students have to complete their work with a personal conclusions that synthesize the contents of the written work. Generally, this section highlights its lack of ability, due to the difficulty of finding and "copy and paste" information to fill this final section. 


\section{Conclusions.}

The use of the Internet both in desktops and mobile computers is really extended between de students on early courses of the degree. They also know and use what we call the Web 2.0, which includes social networks, blogs and other information media.

At the moment of searching information we appreciate that they use Internet mainly as source of information of all kind and content. The habits are quite flat independently from the type of information to search.

The searches are frequently made in Spanish. The students give a lot of credibility to the obtained result. In this respect it may be noted that the selected students from low courses still haven't developed a critical spirit that allows them to select and redact quality information.

From all the previous, we obtain that teachers still have a huge amount of work training the students on these processes. Nowadays, the amount of information that can be transmitted and teach on a degree is quite limited. By the way it is more important to provide these students with the proper tools to search this information that is really relevant form them in a given moment to their studies or professional development. This corollary has even more importance in those disciplines on which the knowledge is in continuous evolution as the technological disciplines.

\section{References}

Abawajy, J.H. \& Hu, M.J. (2005), A new Internet meta-search engine and implementation, ACS/IEEE International Conference on Computer Systems and Applications.

Brandt, D.S, (1997), Constructivism: Teaching for Understanding of the Internet, Communications of the ACM, Vol. 40, $\mathrm{n}^{\circ} 10$.

Boo V. K. \& Anthony, P. (2011), Meta search engine powered by DBpedia, International Conference on Semantic Technology and Information Retrieval (STAIR).

Efthimiadis E. N., (2009), How Students Search for Consumer Health Information on the Web, Hawaii International Conference on System Sciences, pp. 1-8.

J. Garcia, A. Jimeno, H. Mora, et al., (2012), Experiences in Teaching Coordination of Engineering Degrees, Int. Conf. on Education and New Learning Technologies.

Hess B., (1999), Graduate student cognition during information retrieval using the World Wide Web: a pilot study, Computers \& Education, vol. 33, no. 1, 1-13.

Hwang G., Tsai P., Tseng J., Lin C., Tsai C., (2007), Meta-Analyzer: A Web-based Environment for Analyzing Student Information Searching Behaviors, International Conference on Innovative Computing, Information and Control.

Jacobson, T., \& Cohen, L. (1997), Teaching Students to Evaluate Internet Sites, The Teaching Professor.

Johnson, J. (2003), Web Bloopers: 60 Common Web Design Mistakes, and How to Avoid Them, Morgan Kaufmann, San Francisco.

Lytras M. D., Mathkour H., Abdalla H.I., Yáñez-Márquez C., Ordóñez de Pablos P. (2014), The Social Media in Academia and Education Research R-evolutions and a 
Paradox: Advanced Next Generation Social Learning Innovation. The Journal of Universal Computer Science, Vol. 20 (15), pp. 1987-1994.

Mora Mora H., Signes Pont MT., Camps Jordá R., García Chamizo JM., (2009), Learning method based on collaborative assessment performed by the students: an application to computer science, ACM SIGCSE Bulletin, 41 (3).

Mora Mora $\mathrm{H}$ et al., (2014), Management of social networks in the educational process, Computers in Human Behavior, doi:10.1016/j.chb.2014.11.010.

Munro J.\& Kwon R. (1997), Searching the net, PC Mag.

Newsweek Staff, (2010), The perfect Search, Newsweek, vol. 134, no. 13, 71-72. Retrieved from: http://www.newsweek.com/perfect-search-166424.

Palmer E.K., \& Kent J.A., (2007), Do Computer Science Department Websites Meet the Needs of Students?, Proceedings. IEEE SoutheastCon, 333-337.

Perrochon L., (1997), A Quick Tutorial on Searching and Evaluating Internet Resources, IEEE Communications Magazine, Vol. 35, Issue: 6, pp. 142-145.

Pointdexter, S. E \& Heck B .S., (1999), "Using the web in your courses: What can you do? What should you do?”, IEEE Control System Magazine, Vol. 19, Issue 1, pp. 83-92.

Quirós Romero, C. \& Crespo Galán, J. (2007), Information Society and the Spanish presence on the Internet, The Economic Value of Spanish. Technical report (in spanish) University Complutense of Madrid. Retrieved from: http://eprints.ucm.es/9639/1/DT0407.pdf

Song G., Salvendy G. (2003), A framework for reuse of user experience in Web browsing. Behaviour \& Information Technology, Vol. 22, No. 2, pp. 79-90.

Spink, A., Jansen B. J., (2004), Web Search: Public Searching of the Web, Kluwer.

Stenger J. B., Goode J.M, (1998), Improving World Wide Web and Library Research, IEEE Control System Magazine, pp 80-83.

Tang J., Wang, T. \& Wang J. (2009), Measure the Influence of Social Networks on Information Diffusion on Blogspheres, International Conference on Machine Learning and Cybernetics, vol. 6, pp. $3492-3498$.

Tsai M.J., Tsai C.-C., (2003) Information searching strategies in web-based science learning: the role of Internet self-efficacy, Innovations in Education and Teaching International, Vol. 40, No. 1, 3-50.

Zhuhadar L., Yang R., Lytras M.D., (2013), The impact of Social Multimedia Systems on cyberlearners. Computers in Human Behavior, Vol. 29 (2), pp. 378-385.

Uden L., Alderson A., Tearne S., (2001), A Conceptual Model For Learning Internet Searching on the Internet, Annual Hawaii International Conference on System Sciences.

Higinio Mora-Mora received the BS degree in computer science engineering and the BS degree in business studies in University of Alicante, Spain, in 1996 and 1997, respectively. He received the PhD degree in computer science from the University of Alicante in 2003. Since 2002, he is a member of the faculty of the Computer Technology and Computation Department at the same university where he is currently an associate professor and researcher of Specialized Processors Architecture Laboratory. His areas of research interest include computer arithmetic and the design of floating points units and approximation algorithms related to VLSI design. 
Maria Teresa Signes Pont received the BS degree in Computer Science in the Institut National des Sciences Appliquées de Toulouse (France) and Physics in the Universidad Nacional de Educación a Distancia (Spain). She received the $\mathrm{PhD}$ in Computer Science from the University of Alicante in 2005. Since 1996 she is a member of the Faculty of Computer Technology and Computation department at the same university where she is currently an associate professor and researcher of Specialized Processors Architecture Laboratory. Her areas of research interest include computer arithmetic, computational biology and the design of floating point units and approximation algorithms related to VLSI design.

Gregorio de Miguel Casado received a BS degree in Computer Science Engineering (2001), a Master degree in Business Administration (2003) and a Ph.D (2010) from the University of Alicante. Since 2007 he has been Assistant Professor in the Department of Computer Science and Systems Engineering, University of Zaragoza. Among other teaching tasks he has contributed to set up two subjects in the Computer Science Degree specialization in Software Engineering about "Decision Support Systems" and "Requirements Engineering". In addition, he has led a two year project for following the set up of a group of subjects in the specialization on Algorithmics and Computability. 